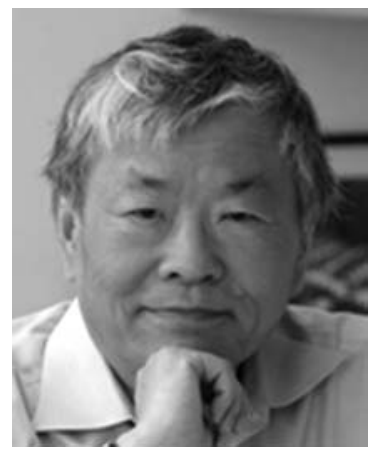

\title{
A Conversation with Susumu Tonegawa
}

\author{
INTERVIEWER: KAREN CARNIOL \\ Deputy Editor, Cell
}

Susumu Tonegawa is the Picower Professor of Biology and Neuroscience at the Massachusetts Institute of Technology (MIT) and the Director of the RIKEN-MIT Center for Neural Circuit Genetics at MIT, as well as the Director of RIKEN Brain Science Institute. He is also an Investigator at the Howard Hughes Medical Institute. Tonegawa won the Nobel Prize in Physiology or Medicine in 1987 for "his discovery of the genetic principle for generation of antibody diversity."

Karen Carniol: Much of your recent work has been focused on memory and in particular on memory engrams.

Dr. Tonegawa: Memory research has a very long history. In the last 100 years, there has been a general idea that when memory information is stored in the brain, the brain cells and the cellular network are altered - biochemically or biophysically - and that those alterations last for a certain period of time corresponding to the length of the memory. However, until recently nobody had pinpointed where exactly a particular memory's information was stored. It's been difficult to study.

There were fragmentary findings in the literature suggesting a synaptic plasticity in which synapse strength was altered or increased, but nobody had shown which cells in the brain had these changes. We wanted to identify the population of cells in which these presumed changes occur, and then to prove that those cells are responsible for the recall of a particular memory. This was very difficult to do until about 2 or 3 years ago, when a technology called optogenetics allowed us to label cells, tag them, and reactivate them to see if their reactivation caused an animal to behave as if it recalled a memory.

With optogenetics, for the first time you can actually see under the microscope the cells that are holding information. In the past, we believed that such cells must exist, in the hippocampus for instance; but the hippocampus has a very heterogeneous population of cells, so we were looking at an averaged picture. Now we can look specifically at the cells which carry memory information. What we see is not a single cell, but a population of cells in the hippocampus which lights up. If we reactivate those cells with an optic fiber implanted deep in the hippocampus, then the animal behaves as if it is recalling a memory. We can even manipulate the cells with optogenetics, and make animals memorize or consolidate memories.

You can also combine such cell manipulation studies with studies of emotion, which has very much to do with memory. A nice experience I had, a terrible experience I had - those kinds of memories are all linked to the brain circuit that controls emotion. The recent work has shown that, in addition to the hippocampus, another area is involved: the amygdala. So we've found that interactions between the hippocampus and amygdala allow us to form memories of different emotional associations.

Though we cannot currently do such optogenetics studies in humans, psychiatrists use a form of psychotherapy that works in a good proportion of depressed patients: They have the patient recall nice, pleasurable experiences they have had in their life. It turns out that in depressed people, memory recall of nice experiences is suppressed, which contributes to the depressed state. Everybody has some nice experiences and some bad experiences in their life, but depressed patients often recall their bad experiences much more easily than their nice experiences.

Empirically, psychiatrists know that helping the patient to recall a nice memory can resolve symptoms in some people, not only for a short time period, but for months, or maybe years. Where in the brain this interaction occurs between the pleasure memory and the fear or anxiety memory is not known. In our most recent work, we are trying to understand the neural circuit for pleasure memory and fear memory, and how one could manipulate these circuits in a mouse in such a way that the positive memory will become dominant.

Karen Carniol: You mentioned that there were two brain areas involved in memory, hippocampus and the amygdala. What's your current interpretation of the functional differences between them in terms of memory coding?

Dr. Tonegawa: The hippocampus has nothing to do with emotion. The primary function of the hippocampus is to store information about the environment and contextwhat we call episodic memory. The amygdala, on the other hand, can encode emotional association - the plus and the minus of an experience. Since the hippocampus and amygdala are connected, they both contribute to memory recall, and thus both good and bad experiences have associations with environment. 
PTSD is an extreme case in which a terrible experience is connected with a specific place or context. A problem for PTSD patients is that they recall the bad memory too easily by encountering an unrelated but similar context, and thus they recall the terrible experience.

We are examining relevant questions in mice by giving them a bad memory and then later giving then a good memory and examining what happened to the bad memory. This we can do by manipulating the memory in both the amygdala and hippocampus. What we find is that there is a circuit which is crucial in switching the dominant emotional asso- ciation of a memory, either positively or negatively. We've also extended this to animals that are chronically stressed. Often chronic stress leads to depression. So now we are making a mouse model of human depression induced by chronic stress. We've found that manipulation of cells in the hippocampus can alleviate symptoms of depression.

The important point is that we can manipulate the emotional state of a mouse by combining the stressed and depressed state with some very positive experiences in different order, and the mouse shows a behavioral impact of the pleasure memory. 


\section{$\$_{\text {CSH }}^{\infty}$ Cold Spring Harbor Symposia SYMPOSIA On Quantitative Biology}

\section{A Conversation with Susumu Tonegawa}

Cold Spring Harb Symp Quant Biol 2014 79: 295-296

Access the most recent version at doi:10.1101/sqb.2014.79.18

\section{License}

Email Alerting Receive free email alerts when new articles cite this article - sign up in Service the box at the top right corner of the article or click here. 